\title{
COMMENTARY
}

\section{Monocyte HLA-DR in sepsis: shall we stop following the flow?}

\author{
Guillaume Monneret ${ }^{1,2^{*}}$ and Fabienne Venet ${ }^{1,2}$ \\ See related research by Cajander et al., http://ccforum.com/content/17/5/R223
}

\begin{abstract}
The best marker for the monitoring of immune alterations in critically ill patients (sepsis, trauma, pancreatitis, surgery, burns) so far remains decreased HLA-DR expression on monocytes measured by flow cytometry as it regularly provides valuable information in terms of mortality prediction or evaluation of risk for secondary infections. As shown by Cajander and colleagues in a recent issue of Critical Care, some promising tools-based molecular biology may circumvent some drawbacks related to flow cytometry. Herein, issues and perspectives about this alternative are discussed.
\end{abstract}

The work by Cajander and colleagues provides preliminary results in quantitation of HLA-DR by real-time polymerase chain reaction as an alternative approach to identify patients presenting with sepsis-induced immunosuppression [1]. Clinical observations and postmortem biopsies have shown that many severe septic patients survive the first critical hours of the syndrome but eventually die in a state of immunosuppression [2]. This immune failure is illustrated by patients' difficulty to fight the primary bacterial infection, decreased resistance to secondary nosocomial infections and reactivation of viral infections usually pathogenic only in immunocompromised hosts. As a consequence, immunostimulatory therapies are now considered an innovative adjunctive strategy for the treatment of sepsis [2]. However, the first critical step is to beforehand identify patients who would actually benefit from these therapies (that is, the most immunosuppressed) [3]. In the absence of

\footnotetext{
* Correspondence: guillaume.monneret@chu-lyon.fr

${ }^{1}$ Hospices Civils de Lyon, Immunology Laboratory, Hôpital E. Herriot, 5 place d'Arsonval, 69437, Lyon, cedex 03, France

'Equipe d'Accueil Mixte 4174 « Hémostase, Inflammation, Sepsis», Hospices Civils de Lyon - Université Claude Bernard Lyon 1, Faculté de Médecine Laennec, 8 rue Guillaume Paradin, 69372, Lyon, cedex 08, France
}

specific clinical signs of their immune response, it is therefore critical to base our judgement on biomarkers reflecting patients' immune status (a missing step in previous clinical trials in the field). This rather pragmatic approach was recently commented on in Critical Care for the importance of monitoring immune functions for putative anti-program death-1 (that is, an immunostimulatory drug) use in patients: 'Nobody would give insulin without glucose monitoring to prevent useless treatment of normoglycaemic or hypoglycaemic patients' [4].

The best marker for the monitoring of immune alterations in critically ill patients so far remains decreased HLA-DR expression on monocytes (mHLA-DR) measured by flow cytometry [3]. The level of HLA-DR expression is an excellent marker of monocyte functionality and anergy (that is, it correlates with decreased antigen presentation capacity and proinflammatory cytokine release). On the clinical side, it is the most frequently assessed marker in various ICU conditions (sepsis, trauma, pancreatitis, surgery, burns, and so forth) and provides valuable information in terms of mortality prediction or evaluation of risk for secondary infections [3]. That given, HLA-DR expression measurement has a couple of drawbacks. First, cell staining should be performed within 2 hours after sampling (can be delayed until 4 hours if blood is stored at $+4^{\circ} \mathrm{C}$ ). This time dependency implies that flow cytometry facilities should be available/present in every hospital. Second, as flow cytometry is not considered a technology for emergency measurements, laboratories using this technique are usually not open 24/7. These two arguments may limit opportunities to conduct studies based on mHLA-DR values.

To circumvent those drawbacks, Cajander and colleagues presently report on measurement of HLA-DR by quantitative reverse transcription polymerase chain reaction (qRT-PCR) as a novel approach to identify immunosuppression in sepsis [1]. The authors established correlations between HLA-DRA/class II transactivator mRNA levels and the monocyte cell surface HLA-DR protein expression measured by flow cytometry. Our 
personal data agree and extend these interesting preliminary results (that is, we performed correlation of six genes coding for different molecules of CMH II complex and mHLA-DR with regard to prediction of mortality in sepsis [5]). Only one point that should be noted regarding the present work is that reported mHLA-DR values measured by flow cytometry are only very slightly decreased (that is, mean 19,000 antibodies bound per cell) while the threshold for normal values is regularly reported above 20,000 antibodies bound per cell [6]. Because values below 10,000 antibodies bound per cell are regularly reported in septic patients, this correlation still needs to be performed for such markedly decreased values as it more appropriately refers to the clinical decision-making values.

Surprisingly, since the pioneering works by Pachot and colleagues [7] and Le Tulzo and colleagues [8] nothing has been published on this aspect while more than 100 articles have dealt with flow cytometry results. This probably highlights the difficulty to translate this transcriptomic approach to a more routine use. Indeed, if major progresses have been made within the last decade regarding the qRTPCR technique in microbiology (quality controls, automated systems, and so forth), no standardised tool for monitoring host response biomarkers is currently available. Although the putative development of automated systems may represent a possible perspective, for now host response mRNA measurement remains based on homemade protocols. Also, no data are available regarding results standardisation (interlaboratory assessment) and analytical specifications (reproducibility, coefficient variation). In line, the question of which reference genes should be used remains an intense matter of debate.

Another issue to minutely decipher is the relative impact of each cell subpopulation expressing HLA-DR as analyses are mostly performed on whole blood (PAXGENE tubes). Therefore, the evaluation of HLA-DR by qRT-PCR often combines expressions from monocytes, dendritic cells, B lymphocytes and activated $\mathrm{T}$ cells whereas flow cytometry specifically measures the monocytic level. In contrast, a standardised flow cytometry protocol for mHLA-DR measurement has been established so as to obtain reproducible results between centres. Pre-analytical requirements were published in 2002 [9]. Subsequently, an international group of experts supervised by the pioneering group from Berlin then defined a consensually accepted standardised protocol in 2005 [6]. Recently, we assessed the robustness of this protocol on fresh whole blood between two centres (Lyon and Grenoble, $100 \mathrm{~km}$ distance between centres) [10]. Blood samples were separated into two aliquots stored at $+4^{\circ} \mathrm{C}$ and then stained and analysed at the same time in two different laboratories on flow cytometers from different companies (Beckman-Coulter,
Hialeah, FL, USA and Becton-Dickinson, San Jose, CA, USA). We observed an excellent correlation between mHLA-DR results [10]. Consequently, mHLA-DR by flow cytometry is ready for multicentric studies.

We therefore come back to our title question: shall we stop following the flow for measuring mHLA-DR? The response is not yet, but we obviously should keep an eye on possible improvements in mHLA-DR measurements by qRT-PCR as this technology is in continuous development and could offer standardised and automated protocols.

\section{Abbreviations}

HLA-DR: Human leukocyte antigen DR; mHLA-DR: HLA-DR expression on monocytes; qRT-PCR: Quantitative reverse transcription polymerase chain reaction.

Published: 06 Jan 2014

\section{References}

1. Cajander S, Bäckman A, Tina E, Strålin K, Söderquist B, Källman J: Preliminary results in quantitation of HLA-DRA by real-time PCR: a promising approach to identify immunosuppression in sepsis. Crit Care 2013, 17:R223.

2. Hotchkiss RS, Monneret G, Payen D: Immunosuppression in sepsis: a novel understanding of the disorder and a new therapeutic approach. Lancet Infect Dis 2013, 13:260-268.

3. Venet F, Lukaszewicz AC, Payen D, Hotchkiss R, Monneret G: Monitoring the immune response in sepsis: a rational approach to administration of immunoadjuvant therapies. Curr Opin Immunol 2013, 25:477-483.

4. Volk HD, Reinke P: To be, or not to be immunocompetent. Crit Care 2013 , 17:185.

5. Cazalis M, Friggeri A, Cavé L, Demaret J, Barbalat V, Cerrato E, Lepape A, Pachot A, Monneret G, Venet F: Decreased HLA-DR antigen-associated invariant chain (CD74) mRNA expression predicts mortality after septic shock. Crit Care 2013, 17:R287.

6. Docke WD, Hoflich C, Davis KA, Rottgers K, Meisel C, Kiefer P, Weber SU, Hedwig-Geissing M, Kreuzfelder E, Tschentscher P, Nebe T, Engel A, Monneret G, Spittler A, Schmolke K, Reinke P, Volk HD, Kunz D: Monitoring temporary immunodepression by flow cytometric measurement of monocytic HLA-DR expression: a multicenter standardized study. Clin Chem 2005, 51:2341-2347.

7. Pachot A, Monneret G, Brion A, Venet F, Bohe J, Bienvenu J, Mougin B, Lepape A: Messenger RNA expression of major histocompatibility complex class II genes in whole blood from septic shock patients. Crit Care Med 2005, 33:31-38. discussion 236-237.

8. Le Tulzo Y, Pangault C, Amiot L, Guilloux V, Tribut O, Arvieux C, Camus C, Fauchet R, Thomas R, Drenou B: Monocyte human leukocyte antigen-DR transcriptional downregulation by cortisol during septic shock. Am J Respir Crit Care Med 2004, 169:1144-1151.

9. Monneret G, Elmenkouri N, Bohe J, Debard AL, Gutowski MC, Bienvenu J, Lepape A: Analytical requirements for measuring monocytic human lymphocyte antigen DR by flow cytometry: application to the monitoring of patients with septic shock. Clin Chem 2002, 48:1589-1592.

10. Demaret J, Walencik A, Jacob MC, Timsit JF, Venet F, Lepape A, Monneret G: Inter-laboratory assessment of flow cytometric monocyte HLA-DR expression in clinical samples. Cytometry B Clin Cytom 2013, 84:59-62.

\section{$10.1186 / \mathrm{cc} 13179$}

Cite this article as: Monneret and Venet: Monocyte HLA-DR in sepsis: shall we stop following the flow?. Critical Care 2014, 18:102 